\title{
A green epoch in the evolutionary history of biological energy sources
}

To the Editor - In an interesting Perspective article, Judson ${ }^{1}$ proposes that the evolution of life may be divided into five epochs, defined by the sequential expansion in available energy sources. Here, I suggest an enhancement to the scheme by considering an additional epoch, acknowledging the status of green plants as the portals through which solar energy enters the terrestrial biosphere. There is persuasive evidence that energy from starchy seeds and underground storage organs profoundly influenced human evolution and, by extension, the consequent course of the Anthropocene. The physiological effects of, and demand for, the glycaemic hit from glucose released by starch hydrolysis are considered to have been critical for the increase in hominid brain size ${ }^{2}$, modifications to the mouth and digestive tract $^{3}$, the use of tools $s^{4}$, the adoption of fire for cooking ${ }^{5}$ and the profound and ongoing socio-cultural developments associated with the rise of agriculture. Starch may have been influential in the evolution of Homo sapiens, but it is only number two in the list of bulk sources of glucose. Half of global biomass is accounted for by cellulose. Humans cannot directly use this repository of sugar but grasslands, abundant sources of cellulosic vegetation, are full of grazers and browsers that can. In this regard, we may view the wild animals and domesticated livestock exploited for meat and milk by hunting and herding as intermediaries employed by humans to access the bioenergy locked up in cellulosic biomass. It's usual to think of meat and dairy products in the modern diet primarily as sources of protein and fat; but in the hierarchy of nutritional priorities, acquiring sufficient calories comes first; and under conditions of subsistence or inanition, when the supply of metabolizable sugars is inadequate or marginal, the carbon skeletons of protein amino acids will be used for bioenergy generation after stripping off the nitrogen in the form of ammonia $^{6}$. It follows that the first settled environments of hunters, trappers, early herders and mixed farmers would tend to have been enriched in nitrogen released by respiration from dietary animal protein. The fertile, disturbed habitats surrounding early settlements and in cultivated fields were an open invitation to synanthropic plants, invading grass crop progenitors with the advantageous traits that we now recognize as the basis of the domestication syndrome ${ }^{7}$. Moving from a starch to a starch + cellulose energy economy would have represented a prodigious increase in the capture of solar photons via photosynthesis and it is reasonable to suppose that this was an important factor in the NeolithicDemographic transition, the period of rapid global population growth between 6,000 and 3,000 years ago $^{8}$. This proposal to interpolate an 'energetic epoch' of starch + cellulose, between those of flesh and fire ${ }^{1}$ is, of course, far from new. It's there in an authoritative, millenia-old publication: 'All flesh is as grass, and all the glory of man as the flower of grass' (King James Bible, Peter 1:24-25).

\author{
Howard Thomas \\ IBERS, Penglais Campus, Aberystwyth University, \\ Aberystwyth SY23 3DA, UK. \\ e-mail:hot@aber.ac.uk
}

Published: 14 August 2017

DOI: 10.1038/s41559-017-0302-8

References

1. Judson, O. P. Nat. Ecol. Evol. 1, 0138 (2017).

2. Hardy, K., Brand-Miller, J., Brown, K. D., Thomas, M. G. \& Copeland, L. Quart. Rev. Biol. 90, 251-268 (2015).

3. Laden, G. \& Wrangham, R. J. Human Evol. 49, 482-498 (2005).

4. Hernandez-Aguilar, R. A. J. Human Evol. 57, 350-364 (2009).

5. Wrangham, R. W. Catching Fire: How Cooking Made Us Human (Basic Books, New York, 2009).

6. Thomas, H. New Phytol. 139, 225-229 (1998).

7. Cunniff, J. et al. PLoS ONE 9, e87586 (2014).

8. Biraben, J. N. Popul. Soc. 394(October), 1-4 (2003).

Competing interests

The author declares no competing financial interests. 